\title{
Bioanalysis
}

\section{High-throughput assay development for combined in vitro toxicity screening of hit compounds and their metabolites in early drug-discovery stage}

\author{
"Looking forward, novel high-throughput, in vitro toxicity screening \\ technologies that combine metabolism will help in crucial toxicity \\ prediction for the ultimate clinical success of a drug."
}

First draft submitted: 28 April 2017; Accepted for publication: 8 May 2017;

Published online: 13 July 2017

Keywords: assay development $\bullet$ drug discovery $\bullet$ high-throughput assay development $\bullet$ hit compounds $\bullet$ In vitro toxicity screening $\bullet$ metabolite toxicity $\bullet$ microdevices

Conventional in vitro toxicity assays for investigational drugs during early discovery stage solely consider the toxicity originating from the parent compound. Toxicity of metabolites is a factor not well studied at this stage and is often left for the latestage development through in vivo tests on animals. Withdrawal of an investigational drug due to metabolism-related adverse effects at later stages of development could have a huge monetary impact. Although animal studies provide valuable information about toxicity originating from drug metabolism, those tests do not always replicate human physiological conditions and are of low throughput. Added to that, a large number of animals involved in the testing and the higher doses used on animals are of ethical concern. Regulatory agencies wish to reduce the number of animals used in toxicity studies. An efficient in vitro assay is highly sought after to eliminate compounds with toxic metabolites early on. In the early discovery stage, the number of compounds dealt with is relatively larger. Therefore, if an early discovery program must incorporate toxicity data from the hit compounds as well as its metabolites, the assay should be high-throughput and cost-effective. Such results should be transferable to humans. Incorporating metabolism into toxicity screening of hit compounds in the early discovery stage is a hot topic of discussion in the drug-discovery community $[1,2]$. The hope is to eliminate surprises and frustration from a drug-discovery program that appeared productive until the last stage.

The reality is that human metabolism is a complex process that involves several metabolic enzymes, multiple tissue involvement and unexpected responses. It is a daunting task and rarely feasible to attempt to incorporate the numerous possible physiological effects in response to an investigational drug or its metabolite, to a functional in vitro assay.

Today, the in vitro toxicity screens that account for metabolism of drug molecules utilize various metabolizing systems such as subcellular, cellular or tissue slices [3]. Each system has its advantages and disadvantages. The question is, could these systems combine toxicity screening of both parent compounds and metabolites to a single system in a highthroughput format? Several new technologies are emerging to make this a reality.

\section{Efforts toward HTS}

Ultrathin films containing both liver metabolizing enzymes (CytP450s) and doublestranded DNA that can generate metabolites of a chemical and detect the DNA damage using electrochemical methods were
Shajila Siricilla

Department of Pharmaceutical Sciences, College of Pharmacy, University of Tennessee Health Science Center, 881 Madison Avenue, Memphis, TN 38163 , USA

Tel.: +1 9014483064

Fax: +1 9014486940

ssiricil@uthsc.edu 
reported [4,5]. Although this approach is a step in the right direction, this is not a whole cell-based assay and will not predict tissue-specific toxicity.

The environmental protective agency (EPA) and other researchers have started to develop novel in vitro assays to include metabolites in the toxicity screening as part of their metabolism challenge program. EPA's efforts are directed toward new technological advances to make high-throughput human-relevant in vitro assays that could combine toxicity screening of both parent compound and its metabolites a reality [6]. Several researchers from academia and industry have proposed forward-looking technologies to do the same. Magnetically immobilized metabolic liver enzymes or enzyme coated magnetic beads loaded in multiwell plates were proposed for use in toxicity screening of investigational drugs in a high-throughput manner. This enzyme-loaded multiwell plates can be used to culture the cell line on which toxicity is to be tested, and the addition of the parent compound to this plate would generate the metabolites. The observed toxic effect will be from both parent compound and its metabolites. Several other scientists have proposed the human liver cell-based multiwell plate assay systems, which would help the extrapolation of the results more accurately to the human clinical subjects. MICRO MT and exogenous xenobiotic metabolism systems do just that. These systems use multiwell plates where liver cell lines are treated with investigational compounds that will be converted to metabolites. The metabolites, along with the parent compounds, are routed through microchannels or porous membranes to neighboring wells containing cell line of interest for toxicity tests. Another system by EPA is working on a simple, cost-effective system, where hepatocytes are gel fixed on a pin-plate lid. These lids can be inserted into a multiwell plate containing cell-line for toxicity screen where metabolites are generated in situ [6]. Primary hepatocytes have been used in toxicity screens where the metabolism mimics human physiology more closely than any other system. New assay devices are trying to incorporate primary hepatocytes in high-throughput screening (HTS) format where incubation with parent compound produces metabolites and then inserted to toxicity screening plates containing cell line of choice. 3D cultures of liver cells and other cell lines such as nerve cell or kidney cells are also being tested for adaptability to HTS devices [6,7]. This approach helps in simultaneous screening of organ-specific toxicities. In addition, novel gene editing technologies that incorporate metabolism associated genes to any cell line of choice will be a great addition to the tools. Microarray 3D bioprinting is another most novel technology explained in EPA's endeavors [6]. 3D, human liver cell cultures are printed in biomimetic hydrogels and can mimic metabolism. The emerging technologies to predict organ toxicities also present novel multiorgan constructs. These are physiologically relevant microdevices that model several organon-a-chip and can mimic human tissue-tissue interactions and allow many combinations of compounds of varying concentrations to be tested [8,9]. If these multidimensional tissue constructs could be incorporated in a regulatory environment, it will be a huge leap in the right direction while traditionally, the cross-organ toxicity was only monitored using animal models.

\section{Conclusion}

Validation of such emerging technologies should be necessary for them to be assuring and to incorporate these assay methodologies to the regulatory drug-discovery scene. It is very crucial to validate the length of period until the metabolizing enzyme activity is maintained in such a system. An overly ambitious future goal could be to incorporate quantification of the metabolite concentration in these HTS systems. Such a quantification of metabolite and remaining parent compound concentrations will validate if those concentrations are physiologically relevant. The challenge would be the incorporation of a suitable analytical method to the HTS assay system [10]. Another question arises, if toxicity is observed for a compound, can these systems be adjusted to differentiate if that toxicity is due to a metabolite or the parent compound by the inclusion of appropriate controls?

Several of these technologies are still under development. However, a nonregulatory toxicity screening with a reliable, cost-effective and fast assay system would highly benefit the early drug-discovery programs to be more efficient. The animal tests should be done to confirm toxicity and should not be used to identify toxicity. Looking forward, novel high-throughput, in vitro toxicity screening technologies that combine metabolism will help in crucial toxicity prediction for the ultimate clinical success of a drug.

\section{Financial \& competing interests disclosure}

The author has no relevant affiliations or financial involvement with any organization or entity with a financial interest in or financial conflict with the subject matter or materials discussed in the manuscript. This includes employment, consultancies, honoraria, stock ownership or options, expert testimony, grants or patents received or pending, or royalties.

No writing assistance was utilized in the production of this manuscript. 


\section{References}

1 Li AP. Screening for human ADME/Tox drug properties in drug discovery. Drug Discov. Today 6(7), 357-366 (2001).

2 Griffini P, James AD, Roberts AD, Pellegatti M. Metabolites in safety testing: issues and approaches to the safety evaluation of human metabolites in a drug that is extensively metabolized. J. Drug Metab. Toxicol. 1, 102 (2010).

3 Coecke S, Ahr H, Blaauboer BJ et al. Metabolism: a bottleneck in in vitro toxicological test development. The report and recommendations of ECVAM workshop 54. Altern. Lab Anim. 34(1), 49-84 (2006).

4 Krishnan S, Hvastkovs EG, Bajrami B, Choudhary D, Schenkman JB, Rusling JF. Synergistic metabolic toxicity screening using microsome/DNA electrochemiluminescent arrays and nanoreactors. Anal. Chem. 80(14), 5279-5285 (2008).

5 Zhou L, Yang J, Estavillo C, Stuart JD, Schenkman JB, Rusling JF. Toxicity screening by electrochemical detection of DNA damage by metabolites generated in situ in ultrathin DNA-enzyme films. J. Am. Chem. Soc. 125(5), 1431-1436 (2003).
6 Erickson BE. Making sure chemical toxicity tests don't miss metabolites. C\&EN 94(32), 16-19 (2016).

7 Gunness P, Mueller D, Shevchenko V, Heinzle E, IngelmanSundberg M, Noor F. 3D organotypic cultures of human HepaRG cells: a tool for in vitro toxicity studies. Toxicol. Sci. 133(1), 67-78 (2013).

8 Kolahchi AR, Mohtaram NK, Modarres HP et al. Microfluidic-based multi-organ platforms for drug discovery. Micromachines 7(162), 1-33 (2016).

9 Bhushan A, Martucci NJ, Usta OB, Yarmush ML. New technologies in drug metabolism and toxicity screening: organ-to-organ interaction. Expert Opin. Drug Metab. Toxicol. 12(5), 475-477 (2016).

10 Ananthula S, Janagam DR, Jamalapuram S, Johnson JR, Mandrell TD, Lowe TL. Development and validation of sensitive LC/MS/MS method for quantitative bioanalysis of levonorgestrel in rat plasma and application to pharmacokinetics study. J. Chromatogr. B 1003, 47-53 (2015). 